\title{
EFFICIENT OPTIMIZATION FOR L-EXTSKY RECOMMENDATIONS
}

\author{
Zhenhua Huang, Juru Wang, Bo Zhang
}

Original scientific paper $L$-extSKY recommendation has recently received a lot of attention in information retrieval community. Literature [1] proposes an algorithm EARG (Efficient Approach based on Regular Grid) to produce the $L$-extSKY objects in one single subspace. However, in multi-user environments, the system generally handles multiple subspace $L$-extSKY recommendations simultaneously. Hence, in this paper, we present an efficient algorithm AOMSR (Algorithm for Optimizing Multiple Subspace $L$-extSKY Recommendations) to remarkably reduce the total response time. Furthermore, we discuss two interesting variations of $L$-extSKY recommendation, i.e., global constraint $L$-extSKY recommendation and local constraint $L$-extSKY recommendation, which are meaningful in practice, and show how our algorithm can be applied for their efficient processing. Detailed theoretical analyses and extensive experiments that demonstrate our solution are both efficient and effective.

Keywords: information retrieval; L-extSKY recommendation; subspace; performance evaluation

Učinkovita optimizacija za L-extSKY preporuke

Izvorni znanstveni članak L-extSKY preporuka je nedavno privukla veliku pažnju pretraživatelja informacija. U literaturi [1] predlaže se algoritam EARG (Efficient Approach based on Regular Grid) za dobivanje $L$-extSKY objekata u jednom jedinom podprostoru. Međutim, u okruženju s više korisnika, sustav obično simultano rješava mnogostruke podprostorne $L$-extSKY preporuke. U ovom radu stoga predstavljamo učinkoviti algoritam AOMSR (Algorithm for Optimizing Multiple Subspace $L$-extSKY Recommendations) u svrhu značajnog smanjenja ukupnog vremena odziva. Nadalje, raspravljamo o dvije interesantne varijacije $L$-extSKY preporuke, tj. globalnom ograničenju $L$-extSKY preporuke i lokalnom ograničenju $L$-extSKY preporuke, koje su od praktičnog značaja i pokazuju kako se naš algoritam može primijeniti u svrhu njihove učinkovite obrade. Detaljna teoretska analiza i velik broj eksperimenata kojima se demonstrira naše rješenje su i efikasni i efektivni.

Ključne riječi: pretraživanje informacija; L-extSKY preporuka; podprostor; ocjena dobivenih rezultata

\section{Introduction}

The skyline recommendation and its computation have attracted much attention recently [2]. To my best knowledge, various techniques have been proposed for subspace skyline recommendation. The existing approaches can be classified in three categories: (1) The first category $[3,4]$ involves the solutions that assume that the recommendation subspace is fixed and use different index structures to improve recommendation performance. (2) Methods in the second category $[5,6]$ consider how to efficiently process all $2^{k}-1$ subspace skyline recommendations. (3) The third category $[7,8]$ tackles the problem of optimizing arbitrary single subspace skyline recommendations. Clearly, if the input dataset is fixed, then the recommendation results returned by these existing approaches will keep invariable.

Literature [1] points out that in most real applications, for a $\zeta$-dimensional dataset $A D$, the cardinality of its recommendation result does not exceed $\left(\ln ^{\zeta-1}|A D|\right) /(|A D| \cdot(\zeta-$ 1)!) of that of $A D$ [9]. So, the recommendation result returned by the existing approaches cannot efficiently assist the users to explore the whole dataset. Motivated by the above fact, the literature [1] extends the semantics of skyline recommendation and proposes a new type of recommendation which is called $L$-extSKY recommendation. Given a set of $\zeta$-dimensional objects, a $L$-extSKY recommendation on the subspace $V(|V| \leq \zeta)$ finds the objects that are dominated by at most $L$ objects on $V$. Conceptually, $K$ represents the thickness of the skylines; the special case $K=0$ corresponds to the conventional skyline recommendation. It is easy to see that compared with the traditional skyline recommendation, the $L$-extSKY recommendation has at least 2 advantages: (1) the $L$-extSKY recommendation can provide more opportunities for users to explore the whole input dataset, since it also considers the non-skyline objects; and (2) the users can flexibly adjust the parameter $L$ to obtain the recommendation result which they need. Consequently, the $L$-extSKY recommendation is more meaningful in practice. Furthermore, the literature [1] presents an algorithm EARG (Efficient Approach based on Regular Grid) to produce the $L$-extSKY objects in arbitrary single subspace. The EARG approach utilizes the regular grid structure and prunes all the cells which are dominated by any other ones, and hence it can evidently reduce the number of comparisons between objects.

However, in multi-user environments, the system generally handles multiple subspace $L$-extSKY recommendations simultaneously. Hence, in this paper, we propose an efficient algorithm AOMSR (Algorithm for Optimizing Multiple Subspace $L$-extSKY Recommendations) to markedly reduce the total recommendation time. The AOMSR algorithm first organizes all issued subspace $L$-extSKY recommendations as a recommendation tree, and then uses the share mechanism in tree paths to improve the total performance of these $L$-extSKY recommendations. Moreover, we discuss two interesting variations of $L$-extSKY recommendation, i.e., global constraint $L$-extSKY recommendation and local constraint $L$ extSKY recommendation, which are meaningful in practice, and show how our algorithm can be applied for their efficient processing. Detailed theoretical analyses and extensive experiments that demonstrate our solution are both efficient and effective. 


\section{The EARG approach}

In this section, we briefly review the EARG approach proposed in the literature [1].

The EARG approach uses the regular grid [10] to index the objects. Consider a set $A D$ of objects which totally has $\zeta$ dimensions. And each dimension $d$ over $A D$ has a set of disjoint ranges which partition the value domain of $d$. Let the extent of each cell on the dimension $d_{i}(1 \leq i \leq \zeta)$ be $\delta_{i}$. Then the cell $c\left[a_{1}, \ldots, a_{\zeta}\right]$ contains all objects with the $i$-th dimension in the range $\left[a_{i} \cdot\left(\delta_{i}-1\right), a_{i}\right.$ $\left.\cdot \delta_{i}\right)$. Conversely, give an object $p$ with attributes $\left(p . x_{1}, \ldots\right.$, $p . x_{\zeta}$ ), its covering cell can be determined (in constant time) as $c\left[b_{1}, \ldots, b_{\zeta}\right]$ where $b_{i}(1 \leq i \leq \zeta)=\left\lfloor p . x_{i} / \delta_{i}\right\rfloor$. Moreover, the EARG approach uses the following four definitions and one theorem, which provide us an opportunity to optimize the performance of the $L$-extSKY recommendation.

Definition 1. Assume that the full space $F$ consists of $\zeta$ dimensions $\left\{d_{1}, \ldots, d_{\zeta}\right\}$, and the subspace $V$ consists of $v$ $(v \leq \zeta)$ dimensions $\left\{d_{1}, \ldots, d_{v}\right\}$.Then if a cell $C(V)=\left[a_{1}, \ldots\right.$, $a_{v}$ ] on $V$ satisfies the following condition, we call $C(V)$ the $V$-dimensional cell of the cell $C(F)=\left[b_{1}, \ldots, b_{v}, \ldots, b_{\zeta}\right]$ on $F: \forall i \in[1, v], a_{i}=b_{i}$.

In order to efficiently realize the EARG approach, the literature [1] distinguishes three possibilities about the relationship between any two $V$-dimensional cells $C_{1}(V)=$ $\left[a_{1}, \ldots, a_{v}\right]$ and $C_{2}(V)=\left[b_{1}, \ldots, b_{v}\right]$.

Definition 2. If two $V$-dimensional cells $C_{1}(V)=\left[a_{1}, \ldots\right.$, $\left.a_{v}\right]$ and $C_{2}(V)=\left[b_{1}, \ldots, b_{v}\right]$ satisfy the following condition, then we say " $C_{1}(V)$ fully dominates $C_{2}(V)$ ": $\forall i \in[1, v], a_{i}<$ $b_{i}$.

For simplicity, literature [1] denotes this relationship as $C_{1}(V) \triangleleft \triangleleft_{V} C_{2}(V)$.

Definition 3. If two $V$-dimensional cells $C_{1}(V)=\left[a_{1}, \ldots\right.$, $\left.a_{v}\right]$ and $C_{2}(V)=\left[b_{1}, \ldots, b_{v}\right]$ satisfy the following condition, then we say " $C_{1}(V)$ partially dominates $C_{2}(V)$ ": $\exists U \subset V$, $\forall i \in[1,|U|], a_{i}=b_{i}$, and $\forall j \in[|U|, v], a_{i}<b_{i}$.

For simplicity, literature [1] denotes this relationship as $C_{1}(V) \triangleleft_{V} C_{2}(V)$.

Definition 4. If two $V$-dimensional cells $C_{1}(V)=\left[a_{1}, \ldots\right.$, $\left.a_{v}\right]$ and $C_{2}(V)=\left[b_{1}, \ldots, b_{v}\right]$ satisfy the following condition, then we say " $C_{1}(V)$ is incomparable with $C_{2}(V)$ ": $\exists i \in[1$, $v], a_{i}<b_{i}$, and $\exists j \in[1, v], b_{j}<a_{j}$.

For simplicity, literature [1] denotes this relationship as $C_{1}(V) \triangleright \triangleleft_{V} C_{2}(V)$.

Theorem 1. Assume that two $V$-dimensional cells $C_{1}(V)=\left[a_{1}, \ldots, a_{v}\right]$ and $C_{2}(V)=\left[b_{1}, \ldots, b_{v}\right]$ cover the objects sets $S_{1}(V)$ and $S_{2}(V)$, respectively. Then we can have:

(a) $\quad C_{1}(V) \triangleleft \triangleleft_{V} C_{2}(V) \Rightarrow \forall p \in S_{1}(V), \quad \forall r \in S_{1}(V), \quad r \quad$ is dominated by $p$;

(b) $C_{1}(V) \triangleleft_{V} C_{2}(V) \Rightarrow \forall p \in S_{1}(V), \quad \forall r \in S_{1}(V), \quad p$ is not dominated by $r$;

(c) $C_{1}(V) \triangleright \triangleleft_{V} C_{2}(V) \Rightarrow \forall p \in S_{1}(V), \forall r \in S_{1}(V), p(r)$ is not dominated by $r(p)$.

Based on the definitions 1-4 and the theorem 1, the EARG approach can be efficiently implemented in the algorithms 1 and 2.

\section{Algorithm 1: EARG}

Input: the set of $\zeta$-dimensional objects $A D$, each object is associated with one counter count whose initial value equals 0 ; the subspace $V$ and its dimensionality $v$; the regular grid index $\Xi(A D, F)$; the parameter $L$.

Output: the $L$-extSKY set $L$-EXSET(AD, $V)$.

Begin

1. $L-E X S E T(A D, V) \leftarrow \varnothing$;

2. Obtain all $V$-dimensional cells which contain at least one object from $\Xi(A D, F)$;

3. Organize these non-empty cells as a sequence $s e q V$ which satisfies: for any two cells $C_{d}(V)=\left[a_{1}, \ldots, a_{v}\right]$ and $C_{\beta}(V)=\left[b_{1}, \ldots, b_{v}\right]$, if $\alpha<\beta$, then $\sum_{i=1}^{v} a_{i} \leq \sum_{i=1}^{v} b_{i}$;

4. For orderly visit each cell $C_{\alpha}(V)$ in $\operatorname{seq} V$ Do

5. $S_{\alpha}(V) \leftarrow$ the set of objects inside $C_{\alpha}(V)$;

6. $\quad C R\left(C_{\alpha}(V)\right) \leftarrow \mathbf{O B C A N S}\left(S_{\alpha}(V), V, L\right)$;

$/ *$ the function $\mathbf{O B C A N S}\left(S_{\alpha}(V), V, L\right)$ returns all candidate $L$-extSKY objects in $S_{\alpha}(V)$, which is shown in Algorithm $2 * 1$

7. flag $\leftarrow$ False;

8. For orderly visit each cell $C_{\lambda}(V)$ in $\operatorname{seq} V$ which Locates before $C_{\alpha}(V)$ Do

9. If $C_{\alpha}(V) \triangleleft \triangleleft_{V} C_{\lambda}(V)$ Then

10. For $\forall p \in C R\left(C_{\alpha}(V)\right)$ Do

11. $\quad$ p.count $\leftarrow$ p.count $+\left|S_{\lambda}(V)\right|$;

12. If $p$.count $\geq L$ Then flag $\leftarrow$ True;

13. $\quad C R\left(C_{\alpha}(V)\right) \leftarrow C R\left(C_{\alpha}(V)\right)-\{p \mid p$.count $>L\}$;

14. If $C_{\alpha}(V) \triangleleft_{V} C_{\lambda}(V)$ Then

15. For $\forall p \in C R\left(C_{\alpha}(V)\right), \forall r \in S_{\lambda}(V)$ Do

16. If $p$ dominates $r$ Then

17. $\quad$. count $\leftarrow$.count +1 ;

18. If $p$.count $\geq L$ Then flag $\leftarrow$ True;

19. $\quad C R\left(C_{\alpha}(V)\right) \leftarrow C R\left(C_{\alpha}(V)\right)-\{p \mid p$.count $>L\}$;

20. If flag $=$ True Then seq $V \leftarrow \operatorname{seq} V-\left\{C_{z}(V) \mid C_{z}(V) \triangleleft \triangleleft_{V}\right.$ $\left.C_{\alpha}(V)\right\}$;

21. $L$-EXSET $(A D, V) \leftarrow L-\operatorname{EXSET}(A D, V) \cup C R\left(C_{\alpha}(V)\right)$;

22. Return $L-E X S E T(A D, V)$;

End

In the step 3 of Algorithm 1, any two cells $C_{\alpha}(V)=$ $\left[a_{1}, \ldots, a_{v}\right]$ and $C_{\beta}(V)=\left[b_{1}, \ldots, b_{v}\right]$ in seq $V$ need to satisfy the condition: $\alpha<\beta \Rightarrow \sum_{i=1}^{v} a_{i} \leq \sum_{i=1}^{v} b_{i}$, which has at least two advantages: (1) If a cell $C_{\alpha}(V)$ is visited earlier, then the probability that it is fully or partially dominated by other cells is lower. (2) If a cell $C_{\alpha}(V)$ locates before another cell $C_{\beta}(V)$, then $C_{\beta}(V)$ cannot fully or partially dominate $C_{\alpha}(V)$, and hence according to Theorem 1, we can have that the objects in $S_{\beta}(V)$ cannot dominate any object in $S_{\alpha}(V)$ on $V$. Consequently, this condition can remarkably reduce the number of comparisons between objects. For each visited cell $C_{\alpha}(V)$, the step 6 uses the function OBCANS which is described in Algorithm 2 to obtain all candidate $L$-extSKY objects in $S_{\alpha}(V)$; that is, if $p \in C R\left(C_{\alpha}(V)\right)$, then $p$ is dominated by at most $K$ objects in $S_{\alpha}(V)$ on $V$. In the steps $8 \div 19$, for each object $p \in$ $C R\left(C_{\alpha}(V)\right)$, the algorithm modifies its counter. And if p.count exceeds $L$ then $p$ can be safely removed from $C R\left(C_{\alpha}(V)\right.$. Specially, the steps $9 \div 13$ focus on considering each cell $C_{\lambda}(V)$ which fully dominates $C_{\alpha}(V)$, while the steps $14 \div 19$ focus on considering each cell $C_{u}(V)$ which partially dominates $C_{\alpha}(V)$. In the steps $9 \div 13$, if $C_{\lambda}(V)$ fully dominates $C_{\alpha}(V)$, then according to Definition 2 , for 
each object $p \in C R\left(C_{\alpha}(V)\right), p$ does not need to be compared with any objects in $C_{\lambda}(V)$, and p.count is directly added $\left|S_{\lambda}(V)\right|$. While in the steps $14 \div 19$, if $C_{u}(V)$ partially dominates $C_{\alpha}(V)$, then according to Definition 3, for each object $p \in C R\left(C_{\alpha}(V)\right), p$ needs to be compared with all objects in $S_{u}(V)$, and p.count is added the number of objects in $S_{u}(V)$ which dominate $p$ on $V$. Furthermore, in the step 20, if the algorithm finds that there exists some object $p \in S_{\alpha}(V)$ whose counter is greater than or equal to $L$, then we can safely remove all cells from seq $V$ which are fully dominated by $C_{d}(V)$.

The function OBCANS utilizes the thought of presorting objects in [4] to obtain $C R\left(C_{\alpha}(V)\right)$, which can be shown in the following algorithm.

\section{Algorithm 2: OBCANS}

Input: the set $S_{\alpha}(V)$ that consists of all objects inside $C_{d}(V)$; the subspace $V$; the parameter $L$.

Output: the set $C R\left(C_{\alpha}(V)\right)$ that consists of all candidate $L$-extSKY objects in $S_{\alpha}(V)$;

\section{Begin}

1. $C R\left(C_{\alpha}(V)\right) \leftarrow \varnothing$;

2. For each object $p \in S_{\alpha}(V)$ Do $p . k e y \leftarrow \sum_{i=1}^{v} \ln (p[i]+1)$;

3. $L_{\alpha}(V) \leftarrow$ the object list obtained by sorting all the objects in $S_{\alpha}(V)$ in the key ascending order;

4. flag $_{1} \leftarrow$ False;

5. For orderly visit each object $p \in L_{\alpha}(V)$ Do

6. flag $_{2} \leftarrow$ False;

7. For orderly visit each object $r \in L_{\alpha}(V)$ which locates before $p$ Do

8. If $p$ dominates $r$ Then

9. $\quad$ p.count $\leftarrow$ p.count +1 ;

10. If p.count $\geq L$ Then flag $_{1} \leftarrow$ True;

11. If p.count $>L$ Then flag $_{2} \leftarrow$ True; break;

12. If flag $_{2}=$ False Then $C R\left(C_{\alpha}(V)\right) \leftarrow C R\left(C_{\alpha}(V)\right) \cup\{p\}$;

13. If flag $_{1}=$ True Then

14. $\quad$ seq $V \leftarrow \operatorname{seq} V-\left\{C_{z}(V) \mid C_{z}(V) \triangleleft \triangleleft_{V} C_{\alpha}(V)\right\}$;

15. Return $C R\left(C_{\alpha}(V)\right)$

End

In the steps $2 \div 3$ of Algorithm 2, we sort the objects in $S_{\alpha}(V)$ using the sorting-operator $\sum_{i=1}^{v} \ln (p[i]+1)$, which has at least three advantages: (1) If an object $p$ in $L_{\alpha}(V)$ is visited earlier, then the probability that it is dominated by other objects in $L_{\alpha}(V)$ on $V$ is lower. (2) The probability that different objects in $L_{\alpha}(V)$ have the same value of $\sum_{i=1}^{v} \ln (p[i]+1)$ is lower. (3) It ensures that any object in $L_{\alpha}(V)$ cannot be dominated by other objects located after it on $V$, and hence can dramatically reduce the number of comparisons between objects. For each object $p \in L_{\alpha}(V)$, in the steps $7 \div 12$, the algorithm modifies p.count. And if p.count does not exceed $L$, then the algorithm adds $p$ into $C R\left(C_{d}(V)\right)$. Furthermore, in the algorithm, we use the Boolean variant flag $_{1}$ as a cell-pruning indicator. When flag $_{1}$ is True, we can know that there exists some object in $C R\left(C_{d}(V)\right)$ whose counter is greater than or equal to $L$. And hence we can safely remove all cells from seqV which are fully dominated by $C_{\alpha}(V)$.

\section{Optimizing multiple subspace $L$-extSKY recommenda- tions simultaneously}

This section focuses on optimizing multiple subspace $L$-extSKY recommendations simultaneously in multi-user environments. Let $u$ be the number of subspace $L$-extSKY recommendations handled in multi-user environments. A naïve solution is to run the EARG approach [1] on the original datasets $u$ times. Obviously, the naïve solution becomes inefficient as the cardinality of original datasets increases, which can be seen in our experimental evaluation. Motivated by this fact, we propose an efficient algorithm AOMSR to reduce the total recommendation time. The AOMSR algorithm first organizes these $u L$ extSKY recommendations as a subspace recommendation tree, and then exploits the share mechanism in tree paths to reduce the total recommendation time. For easily understanding, in the following parts, we use p.count ${ }_{V}$ to denote the counter of $p$ on $V$.

Definition 5. Assume that the list $S Q B$ contains $u$ subspaces $\left\langle V_{0}, \ldots, V_{u}\right\rangle$. Then if $S Q B$ satisfies the following two properties, then we call it a consistent subspace sequence: (1) $V_{0}=\bigcup_{i=1}^{u} V_{i}$; and (2) $\forall V_{i}, V_{j} \in S Q B$, $i<j \Rightarrow\left|V_{i}\right| \geq\left|V_{j}\right|$, where $\left|V_{i}\right|$ is the dimensionality of $V_{i}$.

Definition 6. The subspace recommendation tree $T_{s b}=$ $(N D, E S)$ is built over a consistent subspace sequence $S Q B=<V_{0}, \ldots, V_{u}>$ and needs to satisfy the following properties.

Property 1 (of ND).

(a) $V_{0}$ is the root of $T_{s b}$;

(b) $N D=\left\{V_{i} \mid V_{i} \in S Q B\right\}$.

Property 2 (of $\left\langle V_{i}, V_{j}>\in E S\right.$ ).

(a) $V_{j} \subset V_{i}$;

(b) $\neg \exists V_{g} \in N D, V_{i} \supset V_{g} \wedge V_{g} \supset V_{j}$;

(c) $\neg \exists V_{h} \in N D$, such that $\left\langle V_{i}, V_{j}\right\rangle \in E S$ and $\left\langle V_{i}, V_{j}\right\rangle$ satisfies Property $2(a)$ and $(b)$, and $h<i$.

Definition 7. Let $A D$ be the set of $\zeta$-dimensional objects, and $r t$ be the root node of the subspace recommendation tree $T_{s b}$. And for each non-root node $V$, we assume that $P V$ is its parent node. Then we can recursively define the seed subspace $L$-extSKY set for each node in $T_{s b}$ as follows:

$\operatorname{seed}(V)= \begin{cases}L-E X S E T(A D, r t) & \text { iff } V=r t \\ L-E X S E T(\operatorname{seed}(P V), V) & \text { otherwise. }\end{cases}$

Definition 8. Let $A D$ be the set of $\zeta$-dimensional objects, and $r t$ be the root node of the subspace recommendation tree $T_{s b}$. And for each non-root node $V$, we assume that $P V$ is its parent node. Then based on the seed subspace $L$-extSKY set, we can define the shadow subspace $L$-extSKY set for each node in $T_{s b}$ as follows:

$$
\operatorname{rep}(V)=\left\{\begin{array}{c}
\varnothing ; \quad \text { iff } V=r t . \\
\{p \mid p \in A D-\operatorname{seed}(P V) \wedge \exists r \in \operatorname{seed}(V) \\
\quad \forall z \in V, p[z]=r[z] ; \quad \text { otherwise. }
\end{array}\right.
$$

Theorem 2. Let $A D$ be the set of $\zeta$-dimensional objects, and $r t$ be the root node of the subspace recommendation tree $T_{s b}$. And for each non-root node $V$, we assume 
that $P V$ is its parent node. Then for each node $V$ in $T_{s b}$, we can have:

$L-\operatorname{EXSET}(A D, V)=L-E X S E T(\operatorname{seed}(V) \cup r e p(V), V)$.

Proof: Assume the root node $r t$ is at level 1 in the tree $T_{s b}$. Then, for $r t$, we can have: $L-E X S E T(\operatorname{seed}(r t) \cup r e p(r t)$, $r t)=L-\operatorname{EXSET}(L-\operatorname{EXSET}(A D, r t) \cup \varnothing, r t)=L-\operatorname{EXSET}(A D, r t)$ So, the theorem holds for the root node rt. Below, we focus on each non-root node $V$ and prove that: (1) $L$ $\operatorname{EXSET}(A D, V) \subseteq L-E X S E T(\operatorname{seed}(V) \cup \operatorname{rep}(V), V)$, and (2) $L-$ $\operatorname{EXSET}(\operatorname{seed}(V) \cup \operatorname{rep}(V), V) \subseteq L-\operatorname{EXSET}(A D, V)$. For (1), we prove its correctness by contradiction. Assume there exists an object $p$ such that $p \in L-E X S E T(A D, V)$ and $p \notin L-$ $\operatorname{EXSET}(\operatorname{seed}(V) \cup \operatorname{rep}(V), V)$. Since $p \notin L-E X S E T(\operatorname{seed}(V) \cup$ $\operatorname{rep}(V), V)$, there exists a set $\mathfrak{I}$ such that $|\mathfrak{I}|>L$ and $\forall r \in \mathfrak{I}$, $p$ dominates $r$. And since $\operatorname{seed}(V) \cup \operatorname{rep}(V) \subseteq A D, p \notin L-$ $\operatorname{EXSET}(A D, V)$. This contradicts the assumption that $p \in L$ $\operatorname{EXSET}(A D, V)$. Therefore, $L-E X S E T(A D, \quad V) \subseteq L$ $\operatorname{EXSET}(\operatorname{seed}(V) \cup \operatorname{rep}(V), V)$. For (2), we also prove its correctness by contradiction. Assume there exists an object $p$ such that $p \in L-E X S E T(\operatorname{seed}(V) \cup \operatorname{rep}(V), V)$ and $p \notin$ $L-\operatorname{EXSET}(A D, V)$. From the assumption, we can easily see that there exists some object $q$ such that $q \in A D$ $(\operatorname{seed}(V) \cup \operatorname{rep}(V))$ and $p$ dominates $q$. Since $q \notin \operatorname{seed}(V) \cup$ $\operatorname{rep}(V), q \notin L-E X S E T(\operatorname{seed}(V) \cup \operatorname{rep}(V), V)$. Hence, q.count $>L$. Therefore, we can have: $p$. count $_{V}>L$. That is, $p \notin L-$ $\operatorname{EXSET}(\operatorname{seed}(V) \cup \operatorname{rep}(V), V)$. This contradicts the above assumption that $p \in L-E X S E T(\operatorname{seed}(V) \cup \operatorname{rep}(V), V)$. So then, $L-E X S E T(\operatorname{seed}(V) \cup \operatorname{rep}(V), V) \subseteq L-E X S E T(A D, V)$. based on the above analyses, we can know that the theorem holds.

The AOMSR algorithm is based on Theorem 2 and can be shown below.

\section{Algorithm 3: AOMSR}

Input: the parameter $L$; the set of $\zeta$-dimensional objects $A D$; the regular grid index $\Xi(A D, F) ; u L$-extSKY recommendations whose corresponding subspaces are $V_{1}, \ldots, V_{u}$.

Output: $u$ subspace $L$-extSKY sets $L$-EXSET $\left(A D, V_{1}\right), \ldots$, $L-\operatorname{EXSET}\left(A D, V_{u}\right)$.

Begin

1. $S Q B \leftarrow$ the consistent subspace sequence containing $u$ subspaces;

2. $T_{s b} \leftarrow$ the subspace recommendation tree over $S Q B$;

3. For each node $V$ in $T_{s b}$ Do

4. If $V$ is the root node $r t$ Then

5. $\quad L-\operatorname{EXSET}(A D, r t) \leftarrow \operatorname{EARG}(A D, r t, \Xi(A D, F), L)$;

6. Else

7. $P V \leftarrow$ the parent node of $V$;

8. $\quad \operatorname{seed}(V) \leftarrow$ EARG $(\operatorname{seed}(P V), V, \Xi(A D, F), L)$;

9. $\Delta(V) \leftarrow \operatorname{seed}(V)$;

10. Divide $\operatorname{seed}(V)$ into $m$ subsets $S D_{1}, \ldots, S D_{m}$ which satisfy the following conditions:

(a) $\forall p, r \in S D_{i}, \forall z \in V, p[z]=r[z]$, and

(b) $\forall p \in S D_{i}, r \in S D_{j}, i \neq j \Rightarrow \exists z \in V, p[z] \neq r[z]$;

11. For each $S D_{i}, i \in[1, m]$ Do

12. Select some object $p$ from $S D_{i}$;

13. $S D_{i} . k e y \leftarrow \sum_{z=1}^{v} p[z]$;

14. Organize these $m$ subsets as a list $L D=<S D_{1}, \ldots$,
$S D_{m}>$ which satisfies: $i<t \Rightarrow S D_{i} . k e y \leq S D_{t} \cdot k e y$;

15. For each subset $S D_{i}$ in $L D$ Do

16. Select some object $p$ from $S D_{i}$;

17. $S B_{i} \leftarrow\{q \mid q \in A D-\Delta(P V) \wedge q$ falls inside the covering cell of $p \wedge \forall z \in[1, v], q[z]=p[z]\}$;

18. For each object $q \in S B_{i}$ Do q. count F $_{V}$.count $_{V}$;

19. For each $S D_{t}$ which locates after $S D_{i}$ in $L D$ Do

20. Select some object $r$ from $S D_{t}$;

21. If $r$ dominates $p$ Then

22.

23.

24.

25.

26.

27.

28. $\quad L-E X S E T(A D, V) \leftarrow \operatorname{seed}(V) \cup \operatorname{rep}(V)$;

29. Return $u$ subspace $L$-extSKY sets $L$-EXSET(AD, $\left.V_{1}\right), \ldots, L-\operatorname{EXSET}\left(A D, V_{u}\right)$.

End

In Algorithm 3, after obtaining the subspace recommendation tree $T_{s b}$ (Steps $1 \div 2$ ), the AOMSR algorithm visits each node $V$ and obtains its subspace $L$ extSKY set $L-E X S E T(A D, V)$ in a breadth-first mode (steps $3 \div 28$ ). In the steps 4 and 5 , for the root node $r t$, since its seed subspace $L$-extSKY set is directly obtained from the original dataset $A D$ using the EARG approach, $\operatorname{seed}(r t)=L-\operatorname{EXSET}(A D, r t)$. While for each non-root node $V$, the AOMSR algorithm needs to utilize the property of Theorem 3 to obtain $L-E X S E T(A D, V)$. The basic idea is that the algorithm first obtains the seed subspace $L$ extSKY set $\operatorname{seed}(V)$ in step 8 , and then uses $\operatorname{seed}(V)$ to produce the correct subspace $L$-extSKY set $L$-EXSET(AD, $V)$ in the succeeding steps. In the step 10, the algorithm divides $\operatorname{seed}(V)$ into $m$ subsets and all the objects in the same subsets share the same values on $V$. And in the steps $11 \div 13$, for each subset $S D_{i}$, the algorithm obtains its key $S D_{i}$.key. Then the step 14 organizes these $m$ subsets as a list $L D=<S D_{1}, \ldots, S D_{m}>$ satisfying: $i<t \Rightarrow S D_{i} \cdot k e y \leq S D_{t} . k e y$. Note that for any two subsets $S D_{i}$ and $S D_{t}$ in $L D$, if $S D_{i}$ locates before $S D_{t}$, then the objects in $S D_{t}$ cannot dominate the objects in $S D_{i}$ on $V$. After obtaining the list $L D$, the algorithm orderly processes each subset $S D_{i}$. In the steps $16 \div 17$, the algorithm first randomly selects an object $p$ from $S D_{i}$ and obtains the set $S B_{i}$ which consists of the objects that belong to $A D-\Delta(P V)$ and fall inside the covering cell of $p$ and share the same values on $V$ with $p$. Then in the step 18 , for each object $q \in S B_{i}$, the algorithm set q. count $_{V}$ to be equal to p.count ${ }_{V}$. Since the objects in $S B_{i}$ are added into the shadow subspace $L$-extSKY set $\operatorname{rep}(V)$ and ultimately are incorporated into $L-E X S E T(A D$, $V)$ (steps $27 \div 28)$, the algorithm needs to handle each subset $S D_{t}$ which locates after $S D_{i}$ in $L D$ (steps $19 \div 26$ ). The handling process can be described as follows. The algorithm first randomly selects an object $r$ from $S D_{t}$ and checks if $r$ is dominated by $p$ on $V$. If yes, then the algorithm continues to evaluate the value of $r$.count $t_{V}+\left|S B_{i}\right|$. If $r$. count $t_{V}+\left|S B_{i}\right|$ is greater than $K$, then the algorithm deletes all the objects in $S D_{t}$ from $\operatorname{seed}(V)$ and removes $S D_{t}$ from $L D$; otherwise, for each object $\delta$ in $S D_{t}, \delta$.count $_{V}$ is added $\left|S B_{i}\right|$. 
Example 1. Assume there exists a set of 2dimensional objects $A D=\left\{p_{1}(0.5,4.9), p_{2}(2.2,3.1), p_{3}(9.7\right.$, $2.8), p_{4}(3.9,3.3), p_{5}(5.6,2.4), p_{6}(4.9,2.1), p_{7}(6.5,4.9)$, $\left.p_{8}(3.7,3.7), p_{9}(9.1,2.4), p_{10}(7.0,4.6)\right\}$. And the user Jukie needs two 1-extSKY recommendations whose corresponding subspaces are $V_{1}=\left\{d_{1}, d_{2}\right\}$ and $V_{2}=\left\{d_{2}\right\}$. The AOMSR algorithm first obtains the subspace recommendation tree $T_{s b}: V_{1} \rightarrow V_{2}$, and then orderly processes these two nodes. For $V_{1}$, the AOMSR algorithm invokes the EARG approach to obtain seed $\left(V_{1}\right)$ from $A D$. And $1-\operatorname{EXSET}\left(A D, V_{1}\right)=\operatorname{seed}\left(V_{1}\right)=\left\{p_{1}, p_{2}, p_{4}, p_{8}, p_{5}, p_{6}\right\}$. For $V_{2}$, the AOMSR algorithm first invokes the EARG approach to obtain $\operatorname{seed}\left(V_{2}\right)$ from $\operatorname{seed}\left(V_{1}\right)$, and $\operatorname{seed}\left(V_{2}\right)=$ $\left\{p_{5}, p_{6}\right\}$. Then the AOMSR algorithm divides $\operatorname{seed}\left(V_{2}\right)$ into two subsets $S D_{1}=\left\{p_{5}\right\}$ and $S D_{2}=\left\{p_{6}\right\}$, and organizes these two subsets as a list $L D=<S D_{2}, S D_{1}>$. The AOMSR algorithm orderly handles these two subsets. For $S D_{2}$, the AOMSR algorithm first randomly selects an object (i.e., $p_{6}$ ) from $S D_{2}$, and then obtains the set $S B_{2}=\varnothing$. Since $S B_{2}$ does not contain any object, the AOMSR algorithm is not necessary to process $S D_{2}$ further. For $S D_{1}$, the AOMSR algorithm also first randomly selects an object (i.e., $p_{5}$ ) from $S D_{1}$, and then obtains the set $S B_{1}=\left\{p_{9}\right\}$. Then, the AOMSR algorithm sets $p_{9} \cdot$ count $_{V 2}$ to be equal to $p_{5}$.count $V_{22}$ (i.e., 1) and adds $p_{9}$ into $\operatorname{rep}\left(V_{2}\right)$. Hence, 1$\operatorname{EXSET}\left(A D, V_{2}\right)=\operatorname{seed}\left(V_{1}\right) \cup \operatorname{rep}\left(V_{2}\right)=\left\{p_{5}, p_{6}, p_{9}\right\}$.

\section{Variations of subspace L-extSKY recommendation}

In many real applications, users are only interested in some special data space defined by the constraints. Hence, we only need to return the subspace $L$-extSKY set $L$ $\operatorname{EXSET}(A D, V)$ within this data space. Typically, each constraint is expressed as a range along a dimension and the conjunction of all constraints forms a hyperrectangle in the $v$-dimensional attribute space. Clearly, according to the practical requirements of users, there are two types of recommendations that users might need, which are respectively described in Definitions 9 and 10.

Definition 9. Let $A D$ be the set of $\zeta$-dimensional objects, and $V=\left\{d_{1}, \ldots, d_{2}\right\}$ be the issued subspace. And for each $d_{i} \in V$, users are only interested in the region $\lambda_{i}=\left[B_{i}\right.$, $\left.E_{i}\right]$. Then the global constraint $L$-extSKY recommendation on $V$ returns the set $L-G E X S E T(A D, V, \lambda)=\{p \mid p \in L$ $\left.\operatorname{EXSET}(A D, V) \wedge \forall i \in[1, v], B_{i} \leq p[i] \leq E_{i}\right\}$, where $\lambda=\lambda_{1} \wedge \ldots$ $\wedge \lambda_{v}$.

From Definition 9, we can easily see that $L$-GEXSET $(A D, V, \lambda)$ is the subset of $L-E X S E T(A D, V)$ and consists of the objects which belong to $L-\operatorname{EXSET}(V, A D)$ and fall inside the data space defined by the constraints $\lambda$. For simplicity, we call $L-G E X S E T(A D, V, \lambda)$ global constraint subspace $L$-extSKY set. Furthermore, if $\forall i \in[1, v], B_{i} \leq$ $p[i] \leq E_{i}$, then we say " $p$ satisfies the constraints $\lambda$ " which is denoted as $\lambda(p)$.

Example 2. Returning to Example 1, 1-EXSET(AD, $\left.V_{1}\right)=\left\{p_{1}, p_{2}, p_{4}, p_{8}, p_{5}, p_{6}\right\}$. When the user Jukie gives the constraints $\lambda=\lambda_{1} \wedge \lambda_{2}$ where $\lambda_{1}=[2,4]$ and $\lambda_{2}=[3,4]$. Then, $1-G E X S E T\left(A D, V_{1}, \lambda\right)=\left\{p_{2}, p_{4}, p_{8}\right\}$.

Definition 10. Let $A D$ be the set of $\zeta$-dimensional objects, and $V=\left\{d_{1}, \ldots, d_{2}\right\}$ be the issued subspace. And for each $d_{i} \in V$, users are only interested in the region $\lambda_{i}=\left[B_{i}\right.$, $\left.E_{i}\right]$. Then the local constraint $L$-extSKY recommendation on $V$ returns the set $L-C E X S E T(A D, V, \lambda)=L-E X S E T(S D$, $V)$, where $S D=\left\{p \mid p \in A D \wedge \forall i \in[1, v], B_{i} \leq p[i] \leq E_{i}\right\}$ and $\lambda=$ $\lambda_{1} \wedge \ldots \wedge \lambda_{v}$.

From Definition 10, we can see that $L-C E X S E T(A D, V$, $\lambda$ ) is the subspace $L$-extSKY set which is obtained from the set $S D$ consists of the objects which fall inside the data space defined by the constraints $\lambda$. It is not difficult to see that $L-C E X S E T(A D, V, \lambda) \not \subset L-E X S E T(S D, V)$. For simplicity, we call $L-C E X S E T(A D, V, \lambda)$ local constraint subspace l-SkyRex set.

Example 3. Returning to Example 1, 1-EXSET(AD, $\left.V_{1}\right)=\left\{p_{1}, p_{2}, p_{4}, p_{8}, p_{5}, p_{6}\right\}$. When the user Jukie gives the constraints $\lambda=\lambda_{1} \wedge \lambda_{2}$ where $\lambda_{1}=[8,10]$ and $\lambda_{2}=[2,3]$. Then $1-\operatorname{CEXSET}\left(A D, V_{1}, \lambda\right)=\left\{p_{3}, p_{9}\right\}$.

It is important to note that we can easily obtain the global constraint subspace $L$-extSKY set and the local constraint subspace L-extSKY set by slightly modifying the EARG approach. For the former, we only need to modify the step 21 of EARG: $L-\operatorname{EXSET}(A D, V) \leftarrow L$ $\operatorname{EXSET}(A D, V) \cup\left\{r \mid r \in C R\left(C_{d}(V) \wedge \forall i \in[1, v], B_{i} \leq r[i] \leq E_{i}\right)\right.$. While for the latter, we only need to replace the set $A D$ as the set $S D=\left\{p \mid p \in A D \wedge \forall i \in[1, v], B_{i} \leq p[i] \leq E_{i}\right\}$. Furthermore, generally given the constraints $\lambda, L-G E X S E T(A D, V$ $\lambda) \neq L-C E X S E T(A D, V, \lambda)$. However, the following theorem shows that if the constraints $\lambda$ satisfy the condition $\forall p, r \in A D, \lambda(p) \wedge p$ dominates $r \Rightarrow \lambda(r)$, then $L-G E X S E T$ $(A D, V, \lambda) \neq L-C E X S E T(A D, V, \lambda)$.

Theorem 3. $L$ et $A D$ be the set of $\zeta$-dimensional objects, and $V$ be the issued subspace. And users are only interested in the data space defined by the constraints $\lambda$. Then we can have: $\forall p, r \in A D, \lambda(p) \wedge p$ dominates $r \Rightarrow \lambda(r)$ $\Leftrightarrow L-G E X S E T(A D, V, \lambda) \neq L-C E X S E T(A D, V, \lambda)$.

Proof: For every $z(0 \leq z \leq L)$, we let $L-E X S E T(A D, V)^{(z)}$ be the set of objects in $L-E X S E T(A D, V)$ whose values of counter equal $z, L-G E X S E T(A D, V, \lambda)^{(z)}$ be the set of objects in $L-G E X S E T(A D, V, \lambda)$ whose values of counter equal $z$, and $L-C E X S E T(A D, V, \lambda)^{(z)}$ be the set of objects in $L-C E X S E T(A D, V, \lambda)$ whose values of counter equal $z$. Obviously,

$$
\begin{gathered}
L-\operatorname{EXSET}(A D, V, \lambda)=\bigcup_{z=0}^{L} L-\operatorname{EXSET}(A D, V, \lambda)^{(z)}, \\
L-G \operatorname{EXSET}(A D, V, \lambda)=\bigcup_{z=0}^{L} L-\operatorname{GEXSET}(A D, V, \lambda)^{(z)}, \\
L-C E X S E T(A D, V, \lambda)=\bigcup_{z=0}^{L} L-\operatorname{CEXSET}(A D, V, \lambda)^{(z)} .
\end{gathered}
$$

In the following part, we will prove by induction on the $z$ $(0 \leq z \leq L)$ that $\forall p, r \in A D, \lambda(p) \wedge(p$ dominates $r) \Rightarrow \lambda(r) \Leftrightarrow L$ $\operatorname{GEXSET}(A D, V, \lambda)^{(z)}=L-C E X S E T(A D, V, \lambda)^{(z)}$.

(1) Base Case. For $z=0$, it is easy to see that $L$ $\operatorname{EXSET}(A D, V)^{(0)}$ is the skyline set of $A D$ on $V$. On the other hand, we can have: $L-G E X S E T(A D, V, \lambda)^{(0)}=\lambda(L-$ $\left.\operatorname{EXSET}(A D, \quad V)^{(0)}\right)$, and $L-C E X S E T(A D, \quad V, \lambda)^{(0)}=L-$ $\operatorname{EXSET}(\lambda(A D), V)^{(0)}$. (i) " $\Rightarrow$ ": we are necessary to prove that if $\forall p, r \in A D, \lambda(p) \wedge(p$ dominates $r)$, then $\lambda(L$ $\left.\operatorname{EXSET}(A D, V)^{(0)}\right)=L-\operatorname{EXSET}(\lambda(A D), \quad V)^{(0)}$. Since, $\forall p$, $r \in A D, \lambda(p) \wedge(p$ dominates $r) \Rightarrow \lambda(r)$, for each $p \in A D$, we only need to focus on the objects which dominate $p$ on $V$ and fall inside the data space defined by $\lambda$. And hence, $\lambda\left(L-\operatorname{EXSET}(A D, V)^{(0)}\right)=L-\operatorname{EXSET}(\lambda(A D), V)^{(0)}$. (ii) “ $\Leftarrow$ ": we prove its correctness by contradiction. Assume that when $\lambda\left(L-E X S E T(A D, V)^{(0)}\right)=L-E X S E T(\lambda(A D), V)^{(0)}, \forall p$, $r \in A D, \lambda(p) \wedge(p$ dominates $r) \nRightarrow \lambda(r)$. Since $\lambda(p) \wedge(p$ domi- 
nates $r) \Rightarrow \lambda(r) \Leftrightarrow \neg(\lambda(p) \wedge(p$ dominates $r)) \vee \lambda(r) ; \exists p$, $r \in A D, \lambda(p) \wedge(p$ dominates $r) \wedge \sim \lambda(r)$. Hence, $\exists p, p \in L-$ $\operatorname{EXSET}(\lambda(A D), V)^{(0)} \Rightarrow \exists p, p \notin \lambda\left(L-E X S E T(A D, V)^{(0)}\right)$. This contradicts with the above assumption that $\lambda(L$ $\left.\operatorname{EXSET}(A D, \quad V)^{(0)}\right)=L-\operatorname{EXSET}(\lambda(A D), \quad V)^{(0)}$. Hence, the theorem stands.

(2) Inductive Hypothesis. For $z \leq n, \forall p, r \in A D, \lambda(p) \wedge(p$ dominates $r) \Rightarrow \lambda(r) \Leftrightarrow \lambda\left(L-E X S E T(A D, \quad V)^{(n)}\right)=L-E X S E T$ $(\lambda(A D), V)^{(n)}$.

(3) Inductive Case. For $z=n+1$, we will prove the correctness of the following formula: $\forall p, r \in A D, \lambda(p) \wedge(p$ dominates $r) \Rightarrow \lambda(r) \Leftrightarrow \lambda\left(L-E X S E T(A D, V)^{(n+1)}\right)=L-E X S E T$ $(\lambda(A D), V)^{(n+1)}$. According to the hypothesis: for $z \leq n, \forall p$, $r \in A D, \quad \lambda(p) \wedge(p \quad$ dominates $r) \Rightarrow \lambda(r) \Leftrightarrow \lambda(L-E X S E T(A D$, $\left.V)^{(n)}\right)=L-E X S E T(\lambda(A D), V)^{(n)}$, we have $\forall p, r \in A D, \lambda(p) \wedge(p$ dominates $r) \Rightarrow \lambda(r) \Leftrightarrow \bigcup_{i=1}^{n} \lambda\left(L-\operatorname{EXSET}(A D, V)^{(i)}\right)=\bigcup_{i=1}^{n} L$ $\operatorname{EXSET}(\lambda(A D), V)^{(i)}$. Hence, $\lambda(p) \wedge(p$ dominates $r) \Rightarrow \lambda(r)$ $\Leftrightarrow \lambda\left(L-\operatorname{EXSET}(A D, \quad V)^{(0)}-\bigcup_{i=0}^{n} \operatorname{L-EXSET}(A D, \quad V)^{(i)}\right)=L-$ $\operatorname{EXSET}(\lambda(A D), V)^{(0)}-\bigcup_{i=1}^{n} L-\operatorname{EXSET}(\lambda(A D), V)^{(i)}$. That is, $\lambda(p) \wedge(p$ dominates $r) \Rightarrow \lambda(r) \Leftrightarrow \lambda\left(L-\operatorname{EXSET}(A D, V)^{(n+1)}\right)=L-$ $\operatorname{EXSET}(\lambda(A D), V)^{(n+1)}$.

\section{Experimental evaluation}

This section conducts an empirical study of our methods using the benchmark synthetic datasets. We evaluate the efficiency and the scalability of the proposed methods. Using the data generator [4], we generate two types of synthetic datasets as described in [4]: (a) Independent datasets where the dimension values of the generated objects are uniformly distributed; (b) Anticorrelated datasets where if an object is good in one dimension, it is unlikely to be good in other dimensions. And each object totally has eight dimensions whose data types are 4-type float. Furthermore, in the following experiments, we fix the number of ranges over each dimension to 5. All our experiments are implemented in Java, running on a PC with i5-3210M 2,50 GHz processor and $2 \mathrm{G}$ main memory.

\subsection{Evaluating multiple subspace L-EXTSKY recommenda- tions}

In this subsection, we focus on evaluating the efficiency of our AOMSR algorithm. It is important to note that in order to show the superiority of the AOMSR algorithm, we also evaluate the naïve solution (i.e., running the EARG approach $u$ times) in the experiments where $u$ is the number of subspaces. We implement this set of experiments in the following experimental setting: (1) the cardinality Card of input datasets varies in the range $\left[1 \times 10^{5}, 7 \times 10^{5}\right]$; (2) the parameter $K$ is fixed to 3 ; (3) the dimensionality $\zeta$ of full space is fixed to 8 ; (4) the number of subspaces varies in the range $[20,60]$ (shown in Fig. 1, Fig. 2 and Fig. 3 show the results of experiments for independent datasets and anti-correlated datasets, respectively.

From Fig. 2 and Fig. 3, we can make the following observations:

(1) The AOMSR algorithm evidently outperforms the EARG solution in all cases. This is mainly because the
AOMSR algorithm organizes all needed subspace $L$ extSKY recommendations as a subspace recommendation tree, and then utilizes the share mechanism in tree paths to reduce the total recommendation time. While the EARG solution simply runs the EARG approach $u$ times where $\mathrm{u}$ is the number of subspaces. For example, in Fig. 2b, when the number of subspace is equal to 60 , the recommendation time of the EARG solution exceeds 1951,3 seconds, while the AOMSR algorithm only needs 800,9 seconds. That is, in this case, the recommendation time of the AOMSR algorithm is only about $41,04 \%$ of that of the EARG solution.

\begin{tabular}{|c|c|c|c|c|c|}
\hline \multirow{2}{*}{ the dimensionality of subspace } & \multicolumn{5}{|c|}{ the number of subspaces } \\
\cline { 2 - 7 } & $\mathbf{2 0}$ & $\mathbf{3 0}$ & $\mathbf{4 0}$ & $\mathbf{5 0}$ & $\mathbf{6 0}$ \\
\hline 7 & 1 & 1 & 1 & 1 & 1 \\
\hline 6 & 2 & 3 & 4 & 5 & 6 \\
\hline 5 & 4 & 6 & 8 & 10 & 12 \\
\hline 4 & 8 & 12 & 16 & 20 & 24 \\
\hline 3 & 5 & 8 & 11 & 14 & 17 \\
\hline
\end{tabular}

Figure 1 The relationship between the number of subspaces and the dimensionality of subspace

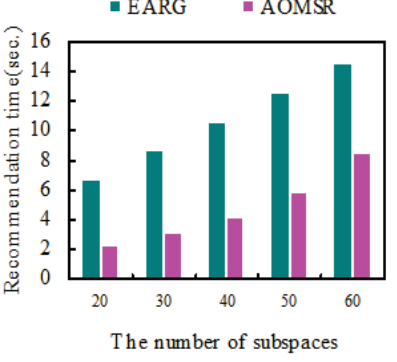

(a) Cardinality $=1 \times 10^{5}$

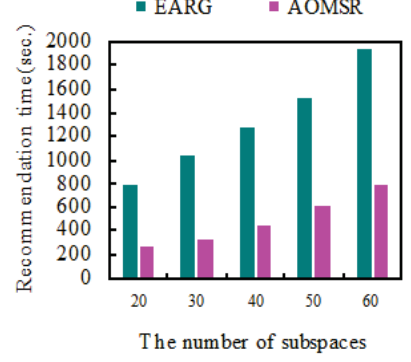

(b) Cardinality $=7 \times 10^{5}$
Figure 2 Independent datasets

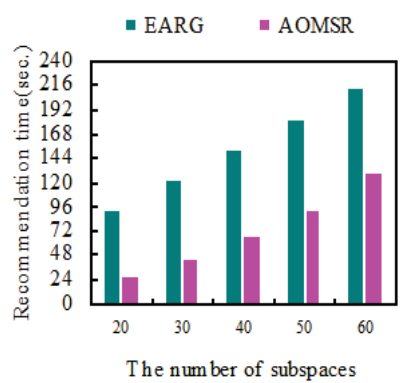

(a) Cardinality $=1 \times 10^{5}$

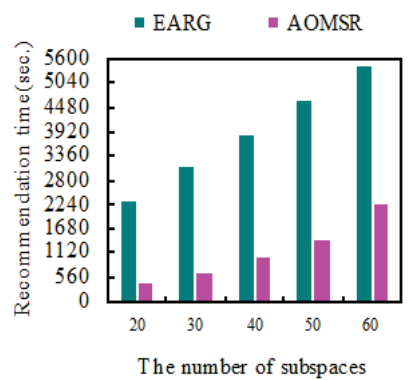

(b) Cardinality $=7 \times 10^{5}$
Figure 3 Anti-correlated datasets

(2) The superiority of the AOMSR algorithm over the EARG solution becomes more marked as the number of subspaces increases. This is mainly because when the number of subspaces increases, the number of $L$-extSKY recommendations which need not to be answered from the original datasets will increase, and hence the sharing effect of the AOMSR algorithm is more evident. For example, in Fig. 3b, when the number of subspaces is equal to 20, the recommendation times of the AOMSR algorithm and the EARG solution are about 469,60 seconds and 2323,29 seconds, respectively. That is, in this case, the AOMSR algorithm only reduces the recommendation time by as much as 1853,69 seconds. While when the number of subspaces is equal to 60 , the recommendation times of the AOMSR algorithm and the EARG solu- 
tion are about 5455,31 seconds and 2262,70 seconds, respectively. That is, in this case, the AOMSR algorithm can reduce the recommendation time by as much as 3192,61 seconds.

(3) The superiority of the AOMSR algorithm over the EARG solution is more marked for anti-correlated datasets than for independent ones. The main reason is that the EARG solution directly runs the EARG approach on original datasets and requires more recommendation time for anti-correlated datasets. For example, in Fig. 2a, when the number of subspace is equal to 50 , the recommendation times of the AOMSR algorithm and the EARG solution are about 5,87 seconds and 12,59 seconds, respectively. That is, in this case, the AOMSR algorithm only reduces the recommendation time by as much as 6,72 seconds. While in Fig. 3a, when the number of subspace is equal to 50, the recommendation times of the AOMSR algorithm and the EARG solution are about 1444,80 seconds and 4672,08 seconds, respectively. That is, in this case, the AOMSR algorithm can reduce the recommendation time by as much as 3227,28 seconds.

\subsection{Evaluating two variations of the subspace $L$-extSKY recommendation}

In this subsection, we focus on evaluating the efficiency of processing our two variations of subspace $L$ extSKY recommendation (i.e., global constraint $L$ extSKY recommendation and local constraint $L$-extSKY recommendation). The compared approaches are G_EARG (L_EARG) proposed in Section 4, and G_SUBSKY (L_SUBSKY) proposed in [7].

We implement this set of experiments in the following experimental setting: (1) the cardinality Card of every dataset varies in the range $\left[1 \times 10^{5}, 7 \times 10^{5}\right]$; (2) the parameter $K$ is fixed to 3 ; (3) the dimensionality $\zeta$ of full space is fixed to 8 ; (4) the dimensionality $v$ of subspace varies in the range $[3,7]$; $(5)$ for each dimension $z$, the constraints $\lambda$ select the region $\left[\min _{z}+\left(\max _{z}-\min _{z}\right) / 5\right.$, $\left.\min _{z}+\left(\max _{z}-\min _{z}\right) \times 4 / 5\right]$ where $\min _{z}$ and $\max _{z}$ are the minimum and the maximum values of objects in every dataset on $z$, respectively. Fig. 4 and Fig. 5 show the results of experiments for independent datasets and anti-correlated datasets, respectively.

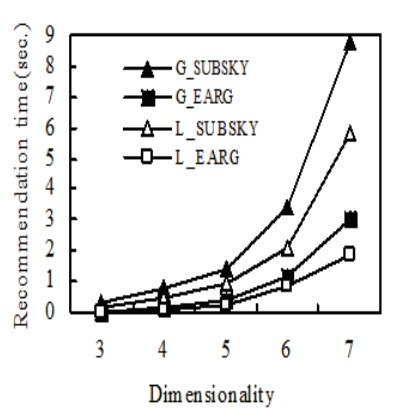

(a) Cardinality $=1 \times 10^{5}$

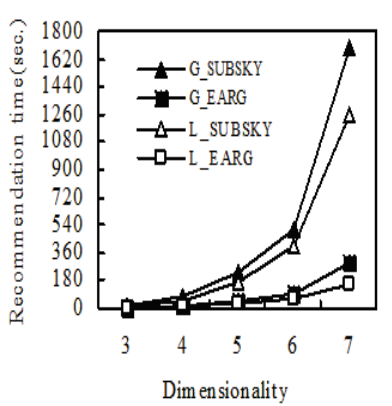

(b) Cardinality $=7 \times 10^{5}$
Figure 4 Independent datasets

From Fig. 4 and Fig. 5, we can observe that the G_EARG and L_EARG respectively outperform the $\mathrm{G}_{-}^{-}$SUBSKY and L_SUBSKY in all cases. This is mainly because the EARG approach evidently outperforms the SUBSKY approach [7] in all cases. For example, in Fig.
$4 \mathrm{~b}$, when the dimensionality of subspace is equal to 7 , the recommendation time of the G_SUBSKY algorithm exceeds 1697,46 seconds, while the G_EARG algorithm only needs 298,17 seconds. That is, in this case, the recommendation time of the G_EARG algorithm is only about $17,57 \%$ of that of the G_SUBSKY algorithm.

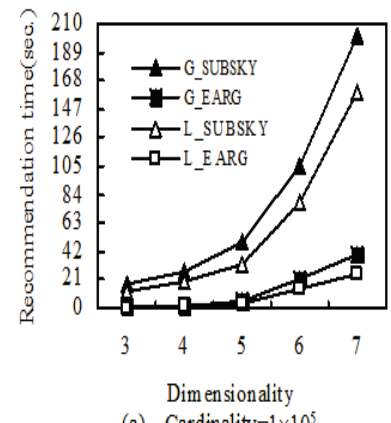

(a) Cardinality $=1 \times 10^{5}$

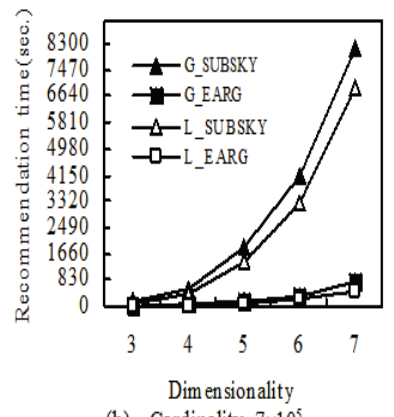

(b) Cardinality $=7 \times 10$
Figure 5 Anti-correlated datasets

\section{Conclusions and future work}

In multi-user environments, the systems generally handle multiple subspace $L$-extSKY recommendations simultaneously. Hence, in this paper, we propose an efficient algorithm AOMSR to evidently reduce the total response time. The AOMSR algorithm first organizes all needed subspace $L$-extSKY recommendations as a subspace recommendation tree, and then employs the share mechanism in tree paths to enhance the total performance. Moreover, we discuss two interesting variations of subspace $L$-extSKY recommendation which are meaningful in practice, and show how our algorithm can be applied for their efficient processing. We also present the detailed predication condition which can cause the equivalence of these two variations. The detailed theoretical analyses and extensive experiments demonstrate that our proposed solution is both efficient and effective.

Future work will focus on using some more efficient index structures to improve the performance of our algorithm, extending our algorithms to stream and P2P environments, and on more experimentation.

\section{Acknowledgements}

This work is supported by the National Natural Science Foundation of China (No. 61272268, 61103069), the Program for New Century Excellent Talents in University (NCET-12-0413), the Fok Ying Tung Education Foundation (No. 142002), the National Basic Research Program of China (973 Program 2014CB340404), and the Fundamental Research Funds for the Central Universities (Tongji University).

\section{References}

[1] Lin, Z. K.; Huang, Z. H.; Xiang, Y. Efficient Processing of k-Quasi Skyline Query. // Systems Engineering -Theory \& Practice. 32, 5(2012), pp. 1098-1106.

[2] Son, W. B.; Hwang, S. W.; Ahn, H. K. MSSQ: Manhattan Spatial Skyline Queries. // Information Systems. 40, 3(2014), pp. 67-83. DOI: 10.1016/j.is.2013.10.001 
[3] Rajasekaran, S.; Zhang, N. On Skyline Groups. // IEEE Transactions on Knowledge and Data Engineering. 26, 4(2014), pp. 942-956. DOI: 10.1109/TKDE.2013.119

[4] Chomicki, J.; Godfrey, P.; Gryz, J.; Liang, D. Skyline with Presorting: Theory and Optimization. // Proceedings of the Int. conference on Intelligent Information Systems / Gdansk, 2005, pp. 595-604. DOI: 10.1007/3-540-32392-9_72

[5] Xia, T.; Zhang, D.; Fang, Z.; Chen, C.; Wang, J. Online Subspace Skyline Query Processing Using the Compressed Skycube. // ACM Transactions on Database Systems. 37, 2(2012), pp. 1-36. DOI: 10.1145/2188349.2188357

[6] Tambaram, K. G.; Lee, J. S.; Rhee, J. W.; Rhee, J. W.; Kang, J. Efficient Skycube Computation Using Point and Domain-based Filtering. // Information Sciences. 180, 7(2010), pp. 1090-1103. DOI: 10.1016/j.ins.2009.11.040

[7] Tao, Y. F.; Xiao, X.; Pei, J. Efficient Skyline and Top-k Retrieval in Subspace. // IEEE Transactions on Knowledge and Data Engineering. 19, 8(2007), pp. 1072-1088. DOI: 10.1109/TKDE.2007.1051

[8] Lee, J.; Hwang, S. Toward Efficient Multidimensional Subspace Skyline Computation. // The VLDB Journal, 23, 1(2014), pp. 129-145. DOI: 10.1007/s00778-013-0317-y

[9] Godfrey, P. Skyline Cardinality for Relational Processing. // Proceedings of the Int. conference on Foundations of Information and Knowledge Systems / Vienna, 2004, pp. 7897. DOI: 10.1007/978-3-540-24627-5_7

[10] Huang, Z.; Xiang, Y.; Zhang, B.; Wang, D.; Liu, X. L. An Efficient Method for K-Means Clustering. // Pattern Recognition and Artificial Intelligence, 23, 4(2010), pp. 516-521.

\section{Authors' addresses}

Zhenhua Huang, PhD.

Department of Computer Science, Tongji University

4800 Cao'an Hwy, Jiading

Shanghai 201804, China

E-mail: huangzhenhua@tongji.edu.cn

Juru Wang, PhD.

Department of Medical Electronics and Information Engineering, Shanghai Medical Instrumentation College

Shanghai 200093, China

E-mail: smic.wangjr@163.com

\section{Bo Zhang, PhD}

Department of Computer Science, Shanghai Normal University Shanghai 200234, China

E-mail: zhangbo.snu@gmail.com 